\section{Estudo transversal sobre fatores associados ao baixo peso ao nascer a partir de informações obtidas em sala de vacinação}

\section{Cross-sectional study of factors associated to low birthweight according to records obtained in vaccination service}

Luiz Fernando Costa Nascimento 1

1 Serviço de Pediatria. Departamento de Medicina. Universidade de Taubaté. Av. Tiradentes, 500. Bom Conselho. Taubaté, SP, Brasil. CEP; 12.030-180

\begin{abstract}
Objectives: to estimate some of the risk factors for low birthweight in Guaratinguetá, a city located in Southeast Brazil, based on records from mothers seeking the vaccination service of the Universal Health Systemin 1998.

Methods: cross-sectional studies with convenience sampling corresponding to 598 mothers interviewed - 28, 9\% of the deliveries in the year of 1998 Independent variables were maternal weight gain, number of medical visits, parity, infection of the genital and urinary tract, arterial hypertension, smoking, mother's age and marriage and job status. Logistic regression was used and relative and population attributable risk estimated; significance level was established at $p<0,05$.

Results: variables with statistical significance present in the final model were maternal age under 20 years old $(O R=2,08)$ other underweight children $(O R=3,94)$, weight gain equal or under $10 \mathrm{~kg}(O R=$ $1,69)$, arterial hypertension $(O R=2,16)$ and smoking $(O R=2,43)$.

Conclusions: the control of these last three factors, that may occur during prenatal care, to the contrary of the other two, may reduce in almost $50 \%$ the prevalence of low birthweight.
\end{abstract}

Key words Infant, low birth weight, Risk factors, Logistic models, Vaccination

\section{Resumo}

Objetivos: estimar alguns fatores de risco para baixo peso ao nascer, em Guaratinguetá, cidade do Sudeste do Brasil, com dados primários obtidos junto a mães que procuraram o setor de vacinação do Sistema Único de Saúde em 1998.

Métodos: estudo transversal com amostra de conveniência e correspondente a 598 mães entrevistadas $28,9 \%$ dos partos ocorridos no ano de 1998. As variáveis independentes foram ganho de peso materno, número de consultas realizadas no pré-natal, paridade, infecção no trato gênito-urinário, hipertensão arterial, outros filhos com baixo peso, tabagismo, idade materna e situações conjugal e trabalhista. Utilizou-se regressão logística e foram estimados os riscos relativos e o risco atribuivel populacional; o nivel de significância foi $p<0,05$.

Resultados: as variáveis com significância estatistica presentes no modelo final foram idade materna inferior a 20 anos $(O R=2,08)$, outros filhos com baixo peso $(O R=3,94)$, ganho de peso igual ou menor que $10 \mathrm{~kg}(O R=1,69)$, hipertensão arterial $(O R=2,16), e$ tabagismo $(O R=2,43)$.

Conclusões: o controle destes três últimos fatores, que pode ocorrer durante o pré-natal ao contrário dos outros dois, pode reduzir em quase $50 \%$ a prevalência de baixo peso ao nascer.

Palavras-chave Recém-nascido de baixo peso, Fatores de risco, Modelos logísticos, Vacinação 


\section{Introdução}

Recém-nascidos com peso menor que $2.500 \mathrm{~g}$ são considerados baixo peso, ${ }^{1}$ fato que pode ser ser devido a uma gestação mais curta, retardo de crescimento intrauterino ou associação desses dois fatores, ${ }^{2}$ além de outros como tabagismo, baixo nível educacional materno, idade materna mais jovem, estado marital, discreto ganho de peso durante a gravidez, hipertensão arterial e infecção do trato genito-urinário na gestação, paridade, menor número de consultas no prénatal e presença de outros filhos com baixo peso ao nascer. $3-5$

O baixo peso ao nascer é provavelmente uma das principais causas, isoladamente, de mortalidade infantil. ${ }^{6}$ Como decorrência do baixo peso ao nascer, podem surgir doenças neurológicas como paralisia cerebral e retardos motor e mental, com baixa performance escolar. 7

A prevalência de baixo peso ao nascer (BPN) oscila entre $3,3 \%$ na Dinamarca 8 a $30 \%$ na Índia. ${ }^{9}$ Entre nós estimam-se valores como $10 \%$ na região $\mathrm{Su}$ deste 10,11 e para o estado de São Paulo, estes valores podem variar entre $6,6 \%$ e $10,4 \% .12$

Para avaliar a prevalência de BPN duas fontes de dados secundários importantes e mais comumente utilizadas são a Declaração de Nascido Vivo12 e o Sistema de Informação sobre Nascidos Vivos (SINASC). 11

Os objetivos do presente trabalho são estimar alguns fatores de risco para o baixo peso ao nascer em crianças de Guaratinguetá, por meio de entrevistas em sala de vacinação.

\section{Métodos}

Guaratinguetá é uma cidade situada no médio Vale do Paraíba paulista, São Paulo, Brasil, com cerca de 100.000 habitantes. Este trabalho é um estudo de prevalência, no qual foram entrevistadas 598 mães que compareceram para vacinar seus filhos no Centro de Saúde de Guaratinguetá, pertencente ao Sistema Único de Saúde, nos meses de setembro e outubro de 1998. Esta amostra foi do tipo de conveniência. O Centro de Saúde é a maior unidade de vacinação da cidade, cujo movimento representa cerca de $90 \%$ do total de vacinas aplicadas, sendo que Guaratinguetá não conta com unidades privadas de vacinação; assim, esta amostra é representativa da população local. O tamanho da amostra foi calculado estimando-se uma prevalência de $10 \%$ de recémnascidos de baixo peso e uma precisão de $3 \%$; o tamanho mínimo obtido foi 384 crianças. As mães responderam a um questionário estruturado, após serem informadas dos objetivos do trabalho e darem seus consentimentos. $\mathrm{O}$ peso ao nascer era o informado verbalmente pela mãe.

Não foram incluídos recém-nascidos gemelares. Foram incluídas crianças com até nove meses de idade, período no qual tomam a vacina anti-sarampo.

A variável dependente neste estudo foi peso ao nascer, obtida de forma contínua categorizando como baixo peso a criança que nasceu com peso inferior a $2.500 \mathrm{~g}$. As variáveis independentes foram: ganho de peso materno na gestação, resultante da diferença entre o peso no início e no final da gestação, que foi categorizado em até $10 \mathrm{~kg}$ inclusive e mais de $10 \mathrm{~kg}$. Consultas no pré-natal categorizadas em até seis consultas e mais de seis consultas; paridade obtida de forma discreta e posteriormente categorizada em um filho e dois ou mais filhos; infecção do trato genito-urinário na gestação presente ou ausente; hipertensão arterial presente ou ausente durante esta gravidez; ocorrência de outros filhos com baixo peso; tabagismo materno durante a gestação, dicotomizado em sim e não; idade materna, dividida em até 19 anos inclusive e maiores que 19 anos; instrução materna, classificada em até oito anos de escolaridade e nove anos ou mais; situação conjugal, se vive maritalmente ou se vive só; situação trabalhista, se está empregada ou não.

Os dados compilados pelo Epi-info 6.01,13 foram analisados para estimar as associações entre as variáveis utilizando-se do teste do qui-quadrado de associação. Posteriormente, utilizou-se o programa Multlr14 para a análise de regressão logística através da técnica forward stepwise. As variáveis que na análise univariada apresentaram $\mathrm{p} \leq 0,20$ foram incluídas no modelo multivariado, sendo que se considerou no modelo final, como significantes, aquelas com $\mathrm{p}<0,05$.

Calcularam-se os odds ratios (OR) para a ocorrência de recém-nascido de baixo peso para cada variável independente. Para se estimar o efeito da redução da incidência de RNBP que poderia ser observada eliminando-se a exposição de toda população ao fator de risco em questão, foi estimado o risco atribuível populacional (RAP) em percentual. Este é a máxima proporção de incidência da doença que pode ser atribuída a um determinado fator. ${ }^{15}$ Foram construídos intervalos de confiança de $95 \%$ e o nível de significância estatística estipulado em $5 \%$.

As variáveis independentes foram rotuladas como fatores de risco para a ocorrência de recémnascido de baixo peso. Alguns totais de respostas são inferiores ao total da amostra por não terem sido respondidas por todas as entrevistadas. 


\section{Resultados}

Foram entrevistadas 598 mães, número acima do sugerido pelo cálculo do tamanho amostral, em virtude da facilidade da obtenção das respostas, e não houve recusa por parte das entrevistadas ou acompanhantes, em participar da pesquisa. O peso de nascimento dos bebês variou entre $1.000 \mathrm{~g}$ e $5.020 \mathrm{~g}$ com média de $3.190 \mathrm{~g}$ e desvio-padrão de $560 \mathrm{~g}$. A idade materna média foi 24,9 anos, com desviopadrão de 5,9 anos. A paridade média encontrada neste estudo foi 1,9 filhos, com desvio-padrão de 0,9 filhos. Foram observados 61 recém-nascidos de baixo peso (RNBP), correspondendo a $10,2 \%$ dos entrevistados. A distribuição segundo o sexo foi de 19 RNBP no sexo masculino $(6,0 \%)$ e 42 RNBP no sexo feminino $(16,4 \%)$, sendo a diferença significativa $(\chi 2=12,71 \mathrm{p}<0,001)$.

A Tabela 1 mostra a análise univariada dos fatores de risco com os respectivos odds ratio (OR), número de expostos na amostra com o percentual e o total de respostas obtidas. Pode ser observado que a presença de fumo na gravidez aumenta a chance de nascimento com baixo peso em quase 2,5 vezes e relato de outros filhos com baixo peso, faz com que esta chance seja quase quatro vezes maior. $\mathrm{O}$ ganho de peso igual ou inferior a $10 \mathrm{~kg}$ aumenta esta chance em $60 \%$. Das mães que participaram deste estudo, cerca de $40 \%$ fumavam e $60 \%$ tinham até oito anos de escolaridade; pouco menos de $75 \%$ destas mães fizeram seis ou mais consultas no pré-natal.

Tabela 1

Análise univariada dos fatores de risco para baixo peso ao nascer com os respectivos odds ratio (OR), intervalo de confiança de $95 \%$ (IC95\%), expostos na amostra e percentagam e total de respostas. Guaratinguetá, SP, Brasil, 1998.

\begin{tabular}{|c|c|c|c|c|c|c|}
\hline \multirow{2}{*}{ Fatores de risco } & \multicolumn{2}{|c|}{ Expostos na amostra } & \multirow{2}{*}{ Total* } & \multirow{2}{*}{ OR } & \multirow{2}{*}{ IC95\% } & \multirow{2}{*}{$\mathbf{P}$} \\
\hline & $\mathbf{n}$ & $\%$ & & & & \\
\hline Consultas no pré-natal menor que seis & 154 & 26,4 & 584 & 1,45 & $1,04-2,00$ & 0,03 \\
\hline Tabagismo na gravidez & 80 & 38,6 & $207 * *$ & 2,46 & $1,27-4,76$ & $<0,01$ \\
\hline Ganho de peso $\leq 10 \mathrm{~kg}$ & 98 & 18,2 & 537 & 1,61 & $1,14-2,32$ & $<0,01$ \\
\hline Infecção urinária & 99 & 17,0 & 582 & 1,29 & $0,64-2,59$ & 0,48 \\
\hline Hipertensão arterial & 86 & 14,7 & 585 & 1,99 & $1,02-3,88$ & 0,04 \\
\hline Instrução materna $\leq 8$ anos & 351 & 59,5 & 590 & 1,27 & $0,82-1,96$ & 0,29 \\
\hline Empregada & 141 & 23,9 & 591 & 0,90 & $0,46-1,76$ & 0,76 \\
\hline Paridade $=1$ & 262 & 45,3 & 578 & 1,06 & $0,88-1,27$ & 0,54 \\
\hline Idade materna $\leq 19$ anos & 122 & 20,7 & 590 & 1,52 & $0,81-2,86$ & 0,19 \\
\hline Vive maritalmente & 485 & 82,8 & 586 & 1,08 & $0,82-1,42$ & 0,59 \\
\hline Outros filhos com BP & 49 & 17,3 & $284 * * *$ & 3,81 & $1,85-7,84$ & $<0,01$ \\
\hline
\end{tabular}

* Total de respostas; ** Mães fumantes; *** Mães com mais de um filho

A Tabela 2 mostra a análise multivariada com as OR ajustadas pelas variáveis contidas neste modelo final, fatores de risco para o baixo peso ao nascer e o RAP para cada fator de risco. As chances de nasci- mento com baixo peso continuam maiores nas mães fumantes e naquelas com relato de outros filhos com baixo peso. 
Odds ratios (OR) ajustados e risco atribuível populacional para as variáveis incluídas no modelo final de regressão logística. Guaratinguetá, SP, Brasil, 1998.

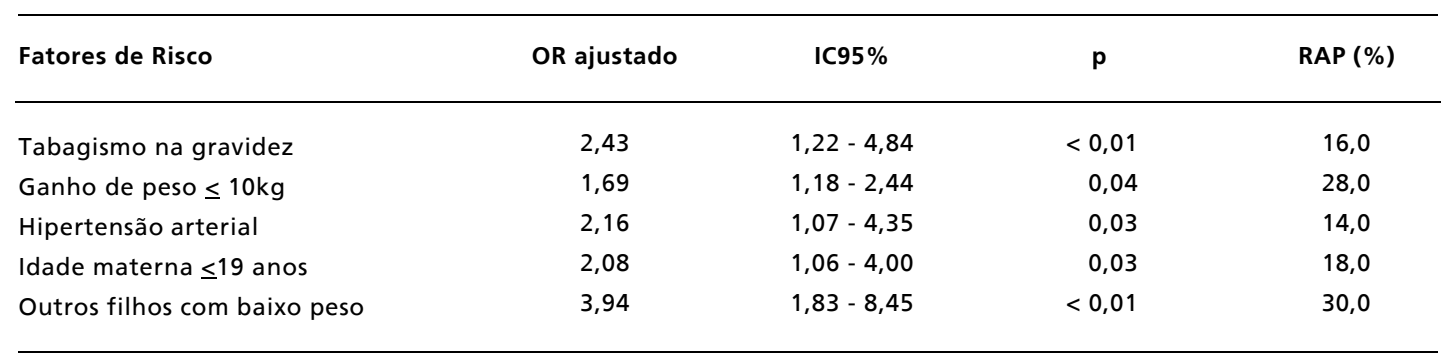

RAP $=$ Risco atribuível populacional; $; \chi^{2}{ }_{5 l}=36,9 p<0,01$

\section{Discussão}

Foram identificados 61 RNBP, o que representa uma prevalência de 10,2\%. Esta prevalência de baixo peso ao nascer está próxima aos resultados encontrados em outros trabalhos realizados no Brasil. Por outro lado, fatores de risco para baixo peso ao nascer que foram identificados nesta amostra, também o foram em outros estudos realizados em nosso país. 5,10-12,16,17 A forma pela qual foram obtidas as informações é que difere de outros trabalhos, visto que os dados costumam ser secundários e, neste estudo, os dados foram obtidos diretamente com as mães. $5,10,11,17,18$

As variáveis deste estudo podem ser agrupadas em variáveis médicas, socioeconômicas e demográficas. Dentre as variáveis médicas estão o número de consultas no pré-natal, o ganho de peso menor ou igual a $10 \mathrm{~kg}$, a presença de infecção urinária e/ou a hipertensão arterial e o tabagismo. A análise univariada mostrou significância estatística para todas, exceto para infecção urinária. Kramer 3 relata que há fracas evidências do papel da infecção urinária na gênese do RNBP e que ela pode ser confundida com a infecção genital, esta sim, com papel importante para o RNBP. A infeção urinária costuma estar associada ao nascimento prematuro. 19

O tabagismo na gestação é um fator de risco para a ocorrência de recém-nascido de baixo peso talvez pela hipóxia intra-útero, levando a uma má nutrição fetal. ${ }^{20}$ A hipertensão arterial, outro fator importante, poderá ser melhor controlada e tratada com uma adequada atenção durante o pré-natal. ${ }^{2} \mathrm{O}$ ganho de peso insuficiente, variável que já foi apontada como fator de risco em outros estudos, também se mostrou importante, 2,5 e pode ser o resultado de um estado nutricional materno comprometido, tendo por causa, possivelmente, uma pobre ingestão de proteínas associada ou não a ingestão deficitária de micronutrientes.4,21 É neste grupo de variáveis que os profissionais de saúde podem promover intervenções visando uma diminuição da prevalência de RNBP.

Dentre as variáveis socioeconômicas estão o grau de instrução materna e a situação trabalhista;5,6 a instrução materna quando conta com até oito anos de escolaridade, que corresponde ao primeiro grau, pode ser causa de desinformação para a mãe e um fator de menor interesse ou de maior dificuldade de acesso a serviços de saúde, em especial o pré-natal; no entanto no presente trabalho não houve significância estatística tanto na análise univariada como no risco relativo; a situação trabalhista empregada, que em princípio pode aumentar a renda familiar, mostrou-se também sem associação significante. Entretanto neste modelo foram incluídas estas variáveis socioeconômicas mesmo que elas perdessem a significância estatística como descrito em outros artigos, em virtude de a associação destas variáveis com RNBP poder ser mediada por outras.

Dentre as variáveis demográficas, a gravidez na adolescência revelou-se estatísticamente significante tanto no modelo univariado como no multivariado; a idade materna está diretamente relacionada às condições sociais e também ao acesso a informações sobre a melhor idade para engravidar, pois a adolescente apresenta um risco maior de ter um recém-nascido de baixo peso até mesmo por imaturidade funcional. 18 Outros filhos com baixo peso foi fator de risco para baixo peso ao nascer neste estudo; esta ocorrência também foi demonstrada e pode também ser causada por fatores genéticos ou por fatores ainda desconhecidos. 2,5 A primiparidade não foi fator de risco; a grande multiparidade, que é fator de risco relatado, 5 não foi analisada neste estudo. 
Finalmente a situação conjugal de viver maritalmente, que poderia ser apresentada como fator protetor pela presença do marido ou companheiro tanto no aspecto psicológico como no econômico, não teve associação estatísticamente significante. Medidas como campanhas educativas e maior facilidade no acesso aos serviços de saúde, poderiam minimizar tanto a gravidez na adolescência como a multiparidade.

A utilização da análise de regressão logística controla os fatores que potencialmente poderiam causar confusão quando se analisam dados dicotômicos isto é, da forma sim/não. Um modelo hierarquizado poderia tratar melhor os determinantes distais como outros filhos com baixo peso e idade materna, e os determinantes proximais como tabagismo na gravidez, hipertensão arterial e ganho de peso na gravidez. 22 Nesta abordagem, poderiam ser estipulados níveis hierárquicos para entrada dos grupos de variáveis, sendo que o grupo das variáveis socioeconômicas pertenceriam ao primeiro nível, posteriormente seriam incluídas as demográficas e finalmente seriam incluídas as variáveis médicas, nesta ordem.

Quanto ao risco atribuível na população, aqueles fatores com maior RAP, devem ser controlados, pois sua redução ou eliminação implicaria numa redução do problema sob o ponto de vista de saúde pública. Nesta amostra, o controle de peso durante a gestação, impedindo um ganho insuficiente, deverá contribuir significativamente para a redução de nascimentos com baixo peso. A atenção à gestante durante o pré-natal dificilmente poderá mudar essa situação mas poderá interferir nos fatores de risco como tabagismo, ganho de peso insuficiente e hipertensão arterial. Quanto às outras situações descritas neste estudo e que apresentaram associações significantes, como outros filhos com baixo peso e idade materna, não passíveis de serem corrigidas neste pré-natal, podem ter sua importância diminuída a médio prazo.

Os vários estudos sobre fatores de risco para o baixo peso ao nascer quase sempre utilizam questionários durante a internação da mãe para o parto, dados se- cundários ou ainda dados obtidos das Declarações de Nascidos Vivo ou ainda do SINASC.5,10,11,17,18

A não inclusão da idade gestacional no rol de variáveis, que pudesse classificar determinada criança com peso inferior a $2.500 \mathrm{~g}$ mas com peso adequado para a idade gestacional, decorre do fato de as mães não se lembrarem com exatidão das informações referentes à última menstruação e também de informações sobre a avaliação feita pelo pediatra na sala de parto na avaliação deste dado. Alguns trabalhos sobre baixo peso não fazem a análise incluindo as variáveis termo e pré-termo. 23,24

Também não se analisaram as crianças que faleceram antes de se fazer a entrevista. Este contingente de crianças provavelmente representa pequeno número na amostra, em virtude da mortalidade infantil em Guaratinguetá ter sido aproximadamente de 20 óbitos por 1.000 nascidos vivos no ano de 1998; como este grupo também pode conter crianças que faleceram mesmo tendo nascido com peso acima de $2.500 \mathrm{~g}$, o estudo não ficou comprometido.

Este trabalho mostra que interrogatórios em salas de vacina, desde que o local tenha um atendimento relevante, tornam tais estudos rápidos e factíveis, pois fornecem dados de maneira menos custosa, visto que as mães têm uma grande capacidade de lembrar dos pesos de nascimento dos filhos, o que torna as informações confiáveis, ${ }^{23}$ evitando-se assim o viés de recordação (recall bias). Por não existirem outras unidades privadas de vacinação, o Centro de Saúde de Guaratinguetá recebe os lactentes de todas as camadas sociais da cidade, não criando um viés de seleção. Outros viéses como o de auto-seleção, quando o entrevistado se oferece para o interrogatório e o de informação não devem ter ocorrido, pois às mães era perguntado se desejavam participar do estudo e as informações não consistiam de respostas elaboradas ou complexas sendo que somente o autor fazia as perguntas. A limitação deste trabalho, do ponto de vista de validade externa, reside no fato deste modelo poder ser aplicado apenas em cidades de médio porte, que não possuam outros centros de vacinação.

\section{Agradecimentos}

O autor agradece a revisão crítica e sugestões apresentadas pelo Prof. Cesar G. Victora, da Universidade Federal de Pelotas, RS, que enriqueceram sobremaneira o trabalho. 


\section{Referências}

1. OMS (Organização Mundial da Saúde). CID-10: classificação estatística internacional de doenças e problemas relacionados à saúde. 10. rev. São Paulo: Ed Universidade de São Paulo; 1998. p.1184

2. Kramer MS. Determinants of low birth weight: methodological assesment and meta-analysis. Bull World Health Organ 1987; 65: 663-737.

3. McDermott S, Coker AL, McKeown RE. Low birth weight and risk of mild mental retardation by ages 5 and 9 to 11 . Paediatr Perinat Epidemiol 1993; 7: 195-204

4. Monteiro MFG, Cervini R. Perfil estatístico de crianças e mães no Brasil: aspectos de saúde e nutrição de crianças no Brasil, 1989. Rio de Janeiro: Fundação Instituto Brasileiro de Geografia e Estatística (FIBGE); 1992.

5. Solla JJSP, Pereira RAG, Medina MG, Pinto LLS. Análisis multifactorial de los factores de riesgo de bajo peso ao nascer em Salvador, Bahia. Rev Panam Salud Publica 1997; 2: 1-6.

6. McCormick NC. The contribution of low birth weight to in fant mortality and childhood morbity. N Eng J Med 1985; 312: 82-90.

7. Paz I, Gale R, Laor A, Danon YL, Stevenson DK, Seidman DS. The cognitive outcome of full-term small-for-gestational-age infants at late adolescence. Obstet Gynecol 1995; 85: 452-6.

8. Olsen J, Frische G. Social differences in reprodutive health Scand J Soc Med 1993; 21: 90-7.

9. Puffer RR, Serrano CV. Patterns of birth weight. Washington, DC: Pan American Health Organization (PAHO); 1987. (Scientific publication, 504).

10. Benicio MH, Monteiro CA, Souza J, Castilho E. Análise multivariada de fatores de risco para baixo peso ao nascer em nascidos vivos do município de São Paulo. Rev Saúde Pública 1985; 19: 311-20.

11. Horta BL, Barros FC, Halpern R, Victora CG. Baixo peso ao nascer em duas coortes de base populacional no sul do Brasil. Cad Saúde Pública 1996; 12 Supl 1: 27-31.

12. Silva AAM, Barbieri MA, Gomes UA, Bettiol H. Trends in low birth weight: a comparison of two birth cohorts separated by 15 - years interval in Ribeirão Preto, Brazil. Bull World Health Organ 1998; 78: 73-84.

13. Dean AG, Dean JA, Coulombier D, Brendel KA, Smith DC, Burton AH, Dicker RC, Sullivan K, Fagan RF, Arner TG. Epi-info version 6: a word processing database, and statistics program for epidemiology onmicrocomputers. Atlanta: Center for Disease Control and Prevention; 1994.
14. Campos-Filho N, Franco EL. A microcomputer program for multiple logistic regression by unconditional and conditional maximun likelyhood methods. Am J Epidemiol 1989; 129: 439-44.

15. Kleinbaun DG, Kupper LL, Morgenstern H. Epidemiological research: principles and quantitative methods. Nova York: Van Nostrand Reinhold; 1982.

16. Michielute R, Ernest JM, Moore ML, Meis P, Sharp PC, Wells HB, Buescher PA. A comparison of risk assessment models for term and preterm low birthweight. Prev Med 1992; 21: 98-109.

17. Costa CEC, Gotlieb SLD. Estudo epidemiológico do peso ao nascer a partir da Declaração de Nascido Vivo. Rev Saúde Pública 1998; 32: 328-34.

18. Maia MAC. Caracterização dos nascidos vivos hospitalares no primeiro ano de implantação do Subsistema de Informação sobre Nascidos Vivos em município de Minas Gerais, Brasil, 1996. Rev Saúde Pública 1997; 31: 5815 .

19. Nascimento LFC. Epidemiology of preterm deliveries in Southeast Brazil: a hospital-based study. Rev. Bras Saúde Matern Infant 2001; 1: 263-8.

20. Mainous AG, Heuston WT. The effect of smoking cessation during pregnancy on preterm delivery and low birth weight. J Fam Pract 1994; 38: 262-6.

21. Rondó PHC, Abbott R, Rodrigues LC, Tomkins AM. The influence of maternal nutritional factors on intrauterine growth retardation in Brazil. Paediatr Perinat Epidemiol 1997; 11: 152-66

22. Victora CG, Huttly SR, Fuchs SC, Olinto MTA. The role of conceptual frameworks in epidemiological analysis: a hierarquial approach. Int J Epidemiol. 1997; 26: 224-7.

23. Walraven GEL, Mkange RJB, van Asten H, van Roosmalen J, van Dongen PWJ, Dolmans WMV. The aetiology of low birthweight in a rural area of Tanzania. Trop Med Int Health 1997; 2: 558-67.

24. Victora CG, Barros FC, Martines JC, Beria JU, Vaughan JP. As mães lembram o peso ao nascer de seus filhos? Rev Saúde Pública 1985; 19: 195-200.

Recebido em 2 março de 2002

Versão final reapresentada em 11 de julho de 2002

Aprovada em 16 de outubro de 2002 